\title{
Growth rate of cosmological perturbations in standard model: Explicit analytical solution
}

\author{
A. D. Chernin ${ }^{1,2,3}$, D. I. Nagirner ${ }^{4,5}$, and S. V. Starikova ${ }^{4}$ \\ 1 Sternberg Astronomical Institute, Moscow University, Moscow, 119899, Russia \\ e-mail: chernin@sai.msu.ru \\ 2 Tuorla Observatory, University of Turku, Piikkiö, 21500, Finland \\ 3 Astronomy Division, Oulu University, Oulu, 90014, Finland \\ ${ }^{4}$ Sobolev Astronomical Institute, St.-Petersburg State University, Univ. pr. 28, Staryi Peterhoff, St.-Petersburg 198504, Russia \\ e-mail: dinmax@dn1756.spb.edu \\ ${ }^{5}$ Isaac Newton Institute of Chile, St. Petersburg Branch
}

Received 6 September 2002 / Accepted 14 November 2002

Abstract. An explicit analytical solution is reported for the growth rate of cosmological perturbations in a flat model with non-zero vacuum energy density.

Key words. cosmology: theory - cosmology: large-scale structure of Universe

\section{Introduction}

The equation for the linear growth rate in the Newtonian theory of cosmological perturbations for nonrelativistic fluid (cold dark matter and baryons) has a form (Zeldovich \& Novikov 1975, Sects. 3 and 4 of Chap. 9; Peebles 1980, Sect. 10)

$\ddot{\delta}+2 H \dot{\delta}=4 \pi G \rho \delta, \quad H=\dot{a} / a$.

Here $\rho$ is the unperturbed cosmological mass density, $\delta$ is the relative density perturbation. The scale factor $a(t)$ (which is also the curvature radius in the non-flat models) is defined by the Friedmann equation:

$\dot{a}^{2}=\frac{8 \pi G}{3} \rho a^{2}+\frac{\Lambda c^{2}}{3} a^{2}-k c^{2}, \quad \rho=\frac{a_{0}^{3}}{a^{3}} \rho_{0}$,

where $a_{0}$ and $\rho_{0}$ are the present values of $a$ and $\rho, \Lambda$ is the cosmological constant, and $k=1,0,-1$ for closed, flat and open models, respectively. The properties of the linear growth of the peArtigle published py ERP Sciences and ayailaple at http: / described in ingreat detail in a review paper by Carroll et al. (1999); see also Lahav et al. (1991), Eisenstein (1997).

The same Eq. (1) governs the evolution of nonlinear perturbations in the Zeldovich (1970) approximation. In this case, a plane perturbation is superimposed on the expansion flow:

$x_{1}=a(t) \chi_{1}+\delta x\left(\chi_{1}, t\right), \quad x_{2}=a(t) \chi_{2}, \quad x_{3}=a(t) \chi_{3}$.

Here $\boldsymbol{x}=\left(x_{1}, x_{2}, x_{3}\right)$ is the Eulerian coordinate (Cartesian) of a fluid element ("particle") with the Lagrangian coordinate $\chi=\left(\chi_{1}, \chi_{2}, \chi_{3}\right)$. Zeldovich (1970) adopted the definition

Send offprint requests to: $\mathrm{A}$. D. Chernin,

e-mail: chernin@sai.msu.ru $\delta=\delta \rho /(\rho+\delta \rho)=-(1 / a) \partial \delta x / \partial \chi_{1}$. The perturbation depends here on one Lagrangian coordinate $\chi_{1}$ and time $t$.

In this paper, a solution to Eq. (1) is presented in an explicit analytical form for a flat model with non-zero $\Lambda$, or vacuum energy density $\rho_{\mathrm{V}}=\Lambda c^{2} /(8 \pi G)$. With the observational discovery of cosmic vacuum (Riess et al. 1998; Perlmutter et al. 1999), this model is becoming the standard one - see recent reviews by Carroll (2000), Weinberg (2000), Sahni \& Starobinsky (2000), Chernin (2001), Peebles \& Ratra (2002).

\section{General solution in integral representation}

The general solution for $a(t)$ is well-known in the integral form:

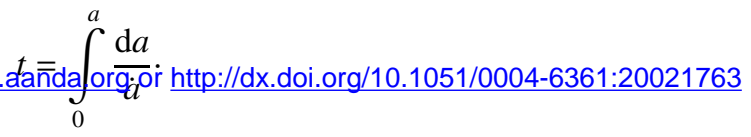

The two linearly independent solutions of Eq. (1) are also known in integral representation (Heath 1977); in the notations used by Peebles (1980) and with the constant factors that make the functions dimensionless, one has:

$\delta_{1}(t)=\frac{\left(\Lambda c^{2}\right)^{5 / 6} a_{0}^{2}}{3 \sqrt{3}} \delta_{2}(t)\left(8 \pi G \rho_{0}\right)^{2 / 3} \int_{0}^{a(t)} \frac{\mathrm{d} a}{\dot{a}^{3}}$,

$\delta_{2}(t)=\frac{1}{\alpha} \frac{\dot{a}(t)}{a(t)}, \quad \alpha=\sqrt{\frac{\Lambda}{3}} c$. 
The solution of Eq. (4) can be expressed in terms of elementary functions and dimensionless integrals that depend on one dimensionless argument and one number parameter:

$\alpha t=J_{1 / 2}(y, A), J_{v}(y, A)=\int_{0}^{y}\left(\frac{y^{\prime}}{Y\left(y^{\prime}, A\right)}\right)^{v} \mathrm{~d} y^{\prime}$.

Here

$Y(y, A)=1+A y+y^{3}, y=\left(\frac{\Lambda c^{2}}{8 \pi G \rho_{0}}\right)^{1 / 3} \frac{a}{a_{0}}$,

$A=-\frac{3 k}{a_{0}^{2}} \Lambda^{-1 / 3}\left(\frac{c^{2}}{8 \pi G \rho_{0}}\right)^{2 / 3}$.

In these notations, one has for the two modes of perturbations:

$\delta_{1}(t)=\frac{Y^{1 / 2}(y, A)}{y^{3 / 2}} J_{3 / 2}(y, A), \quad \delta_{2}(t)=\frac{Y^{1 / 2}(y, A)}{y^{3 / 2}}$.

It is worthwhile to mention some basic properties of the perturbation modes.

1. As it is seen from (10), in the limit $t \rightarrow 0$, the solutions are given by the power-law functions: $y \sim(3 \alpha t / 2)^{2 / 3} \rightarrow 0, \delta_{1} \sim$ $2 y / 5 \sim 2(3 \alpha t / 2)^{2 / 3} / 5, \delta_{2} \sim y^{-3 / 2} \sim 2 /(3 \alpha t) \rightarrow \infty$. In this limit, the dynamical effect of cosmic vacuum is negligible, and the solutions are asymptotically the same as in the theory of linear perturbations with zero $\Lambda$ (Lifshits 1946).

2 . For $k>0$ (close model) and $A<-3 \cdot 2^{-2 / 3}$, the polynomial $Y(y, A)$ has a positive root $y_{*}<2^{-1 / 3}$. In this case, the scale factor is limited: $a \leq a_{*}=a_{0} y_{*}\left(\frac{8 \pi G \rho_{0}}{\Lambda c^{2}}\right)^{1 / 3}$. The maximal value $a=a_{*}$ is reached at $t_{*}=(1 / c \sqrt{\Lambda}) J_{1 / 2}\left(y_{*}, A\right)$. At this time moment, $\delta_{1}=2 y_{*} /\left(2 y_{*}^{3}-1\right), \delta_{2}=0$.

3 . In opposite case $A>-3 \cdot 2^{-2 / 3}$, the expansion continues infinitely. Then $\delta_{1}$ goes to $J_{3 / 2}(\infty, A)$ and $\delta_{2} \rightarrow 1$, when $a$ goes to $\infty$. The same is in an open model $(k<0)$ in which the expansion is always infinite. $2^{-1 / 3}$

4. If $k>0$ and $A=-3 \cdot 2^{-2 / 3}$, then $\delta_{1} \rightarrow \infty$ with $y \rightarrow y_{*}=$

5. If $A>-3 \cdot 2^{-2 / 3}$, the integral $J_{3 / 2}(\infty, A)$ converges and there exists a special solution

$\delta_{3}(t)=\delta_{2}(t)\left[J_{3 / 2}(\infty, A)-J_{3 / 2}(y, A)\right]$.

In this solution, $\delta_{3} \propto \delta_{2}$ when $y \rightarrow 0$, and $\delta_{3} \sim 1 /\left(2 y^{2}\right)$ when $y \rightarrow \infty$. If $A \leq-3 \cdot 2^{-2 / 3}$, such a solution does not exist. For more about the classification of the solutions see Bildhauer et al. (1992).

\section{Explicit solution for flat model}

In a flat model $(k=0)$, the solutions for both unperturbed model and perturbations can be expressed in terms of standard functions. Indeed, the solution of Eqs. (7) and (2) is

$y=\sinh ^{2 / 3}\left(\frac{3}{2} \alpha t\right), \quad a(t)=a_{0}\left(\frac{8 \pi G \rho_{0}}{3 \alpha^{2}}\right)^{1 / 3} y$.

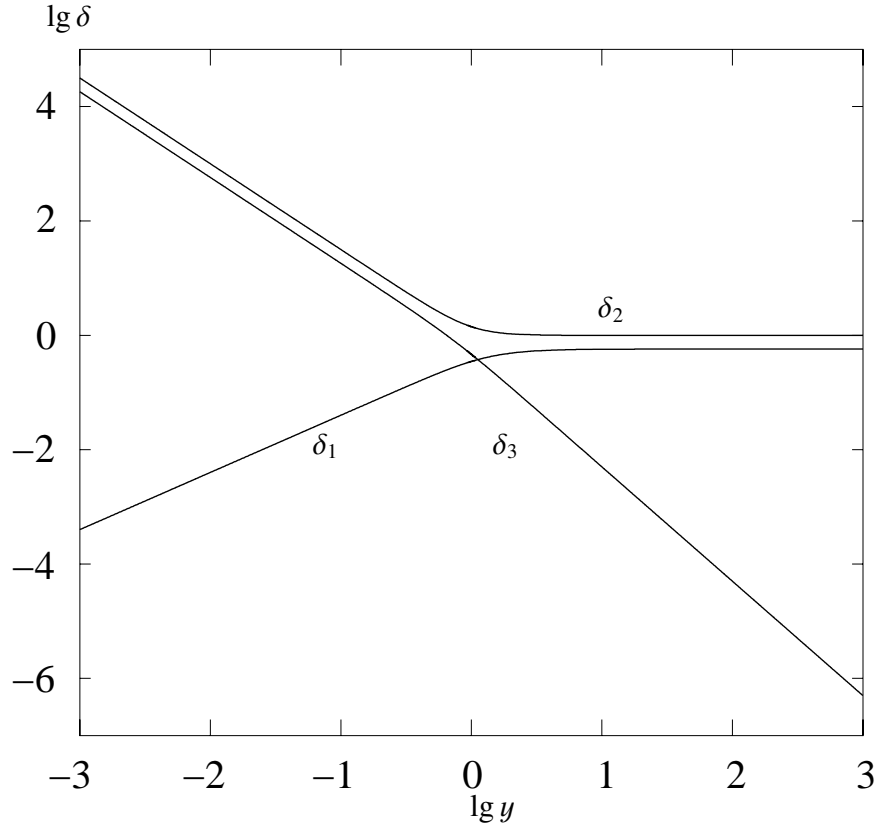

Fig. 1. The perturbations $\delta_{1}(t), \delta_{2}(t)$ and $\delta_{3}(t)$ as functions of $y$.

Then one has for the perturbations:

$\delta_{2}(t)=\frac{\sqrt{1+y^{3}}}{y^{3 / 2}}, \quad \delta_{1}(t)=\delta_{2}(t) J_{3 / 2}(y, 0)$.

The first mode here, $\delta_{1}(t)$, is given in the terms of hypergeometric functions:

$\delta_{1}(t)= \begin{cases}\frac{2}{5} y F\left(1, \frac{1}{3}, \frac{11}{6} ;-y^{3}\right) & \text { if } 0 \leq y<1, \\ \frac{2}{5} \frac{y}{1+y^{3}} F\left(1, \frac{3}{2}, \frac{11}{6} ; \frac{y^{3}}{1+y^{3}}\right) & \text { if } y \sim 1, \\ c_{0} \delta_{2}(t)-\delta_{3}(t) & \text { if } y>1,\end{cases}$

where $c_{0}=\Gamma(5 / 6) \Gamma(5 / 3) / \sqrt{\pi}$ and

$\delta_{3}(t)=\frac{1}{2 y^{2}} F\left(1, \frac{1}{6}, \frac{5}{3} ;-\frac{1}{y^{3}}\right)=c_{0} \delta_{2}(t)-\delta_{1}(t)$.

Here the hypergeometric function is represented via three convergent hypergeometric series in the three different regions of the variable $y$.

In Bildhauer et al. (1992), the solution $\delta_{1}$ is expressed in terms of incomplete beta-function.

Figure 1 demonstrates the behaviour of $\delta_{1}, \delta_{2}$ and $\delta_{3}$ as functions of $y$ which is the dimensionless scale factor normalized according to Eq. (12).

The time behaviour of the three perturbation modes is fairly simple in this log-log plot. The functions $\delta_{1}$ and $\delta_{2}$ go to constants at $y \gg 1$. At the opposite limit $y \ll 1$, they have the correct (see Sect. 2) power-law asymptotes: $\delta_{1} \propto y, \delta_{2} \propto y^{-3 / 2}$. The special mode $\delta_{3}$ scales like $\delta_{2}$ at $y<1$ and it is somewhat steeper at $y>1$.

\section{Conclusions}

The solution of Sect. 3 demonstrates the growth rate of cosmological perturbations in an explicit analytical form for the standard model with zero spatial curvature and non-zero vacuum 
Table 1. Asymptotic behaviour of $\delta_{i}(t)\left[1+\frac{\mathrm{d} \ln \delta_{i}}{\mathrm{~d} \ln y}\right]$.

\begin{tabular}{ccc}
\hline \hline & $t \rightarrow 0$ & $t \rightarrow+\infty$ \\
\cline { 2 - 3 } Mode & $y \sim(3 \alpha t / 2)^{2 / 3} \times$ & $y \sim 2^{-2 / 3} \mathrm{e}^{\alpha t} \times$ \\
& $\times\left(1+\frac{1}{4} \alpha^{2} t^{2}\right)$ & $\times\left(1-\frac{2}{3} \mathrm{e}^{-3 \alpha t}\right)$ \\
\hline 1 & $\frac{4}{5} y\left(1-\frac{5}{11} y^{3}\right)$ & $c_{0}+\frac{1}{2} y^{-2}$ \\
2 & $-\frac{1}{2} y^{-3 / 2}\left(1-\frac{5}{2} y^{3}\right)$ & $1-y^{-3}$ \\
3 & $-\frac{c_{0}}{2} y^{-3 / 2}-\frac{4}{5} y$ & $-\frac{1}{2} y^{-2}\left(1-\frac{2}{5} y^{-3}\right)$ \\
\hline
\end{tabular}

energy density $\rho_{\mathrm{V}}$. The solution describes the evolution of weak linear perturbations of an arbitrary spatial shape. According to $(1)$,

$\delta(\chi, t)=f_{1}(\chi) \delta_{1}(t)+f_{2}(\chi) \delta_{2}(t)$

where $f_{1}(\chi)$ and $f_{2}(\chi)$ are arbitrary functions of the three Lagrangian coordinates. The special case $\delta(\chi, t)=f_{3}(\chi) \delta_{3}(t)$ corresponds to the restriction $c_{0} f_{1}=-f_{2}=-c_{0} f_{3}$.

The solution of Sect. 3 is also valid for the evolution of nonlinear planar perturbations in the Zeldovich approximation:

$\delta x=a(t)\left[F_{1}\left(\chi_{1}\right) \delta_{1}(t)+F_{2}\left(\chi_{1}\right) \delta_{2}(t)\right]$.

Here $F_{i}^{\prime}\left(\chi_{1}\right)=-f_{i}\left(\chi_{1}\right), i=1,2,3$, since in Eq. (17) the functions $F_{i}$ can depend on one Lagrangian coordinate only. The third solution appears, if $c_{0} F_{1}=-F_{2}=-c_{0} F_{3}$.

The planar perturbation velocity field $\delta v_{1}$ of the solution of Eq. (17) is superimposed on the Hubble velocity field $\boldsymbol{v}=\dot{a}(t) \chi$. One has for each of the modes: $\delta v_{1}^{(i)}=$ $F_{i}\left(\chi_{1}\right)\left[\dot{a}(t) \delta_{i}(t)+a(t) \dot{\delta}_{i}(t)\right]$. The relative velocity perturbation

$\frac{\delta v_{1}^{(i)}}{v_{1}}=\frac{\delta v_{1}^{(i)}}{\dot{a}(t) \chi_{1}}=\frac{F_{i}\left(\chi_{1}\right)}{\chi_{1}} \delta_{i}(t)\left[1+\frac{\mathrm{d} \ln \delta_{i}}{\mathrm{~d} \ln y}\right]$.

In Table 1, the asymptotes of $\frac{\delta v_{1}^{(i)}}{v_{1}} \frac{\chi_{1}}{F_{i}\left(\chi_{1}\right)}$ are given for the limits $t \rightarrow 0$ and $t \rightarrow \infty$.
In both linear and nonlinear cases, the solution describes the development of gravitational instability in cold dark matter on the cosmic vacuum background. The instability is terminated near $y=1$, or the redshift $z \simeq z_{\mathrm{V}}=\left(2 \rho_{\mathrm{V}} / \rho_{0}\right)^{1 / 3}-1$, when the vacuum antigravity begins to dominate dynamically over the selfgravity of matter. A similar termination of the instability is known in an open model with no cosmological constant (Peebles 1993). In the open model, perturbations become also "frozen in" the expansion flow, in the limit of an inertial $(a \propto t)$ expansion in vacuum of zero energy. Cosmic vacuum of non-zero energy leads to the exponentially rapid freeze-out of the perturbation modes $\delta_{1}$ and $\delta_{2}$ soon after $z_{\mathrm{V}}$. As for the special mode $\delta_{3}$, it remains falling after $z_{\mathrm{V}}$.

Acknowledgements. D.N. and S.S. appreciate a partial support from the Russian Leading Scientific Schools grant 00-15-96607 and Federal Program "Integration", project B0029.

\section{References}

Bildhauer, S., Buchert, T., \& Kasai, M. 1992, A\&A, 263, 23

Carrol, S. M. 2000 [astro-ph/0004075]

Carrol, S. M., Press, W. H., \& Turner, E. L. 1999, ARA\&A, 30, 499

Chernin, A. D. 2001, Physics-Uspekhi, 44, 1099

Eisenstein, D. J. 1997 [astro-ph/9709054]

Heath, D. J. 1977, MNRAS, 179, 351

Lahav, O., Lilje, P. B., Primack, J. R., \& Rees, M. J. 1991, MNRAS 251,128

Lifshits, E. M. 1946, JETP, 16, 587

Peebles, P. J. E. 1980, The Large Scale Structure of the Universe (Princeton University Press, Prinsceton)

Peebles, P. J. E. 1993, Principles of Physical Cosmology (Princeton University Press, Princeton)

Peebles, P. J. E., \& Ratra, B. 2002 [astro-ph/0207347]

Perlmutter, S., Aldering, G., Goldhaber, G., et al. 1999, ApJ, 517, 565

Riess, A., Filipenko, A. V., Challis, P., et al. 1998, AJ, 116, 1009

Sahni, V., \& Starobinsky, A. 2000, IJMP, 9, 373

Weinberg, S. 2000 [astro-ph/0005265]

Zeldovich, Ya. B. 1970, A\&A, 5, 84

Zeldovich, Ya. B., \& Novikov, I. D. 1975, The Structure and Evolution of the Universe. Nauka, Moscow. (University of Chicago Press, Chicago, 1983) 